\title{
Diffusion-induced instability and chaos in random oscillator networks
}

\author{
Hiroya Nakao $^{1}$ and Alexander S. Mikhailov ${ }^{2}$ \\ ${ }^{1}$ Department of Physics, Kyoto University, Kyoto 606-8502, Japan \\ ${ }^{2}$ Abteilung Physikalische Chemie, Fritz-Haber-Institut der Max-Planck-Gesellschaft, Faradayweg 4-6, 14195 Berlin, Germany
}

(Received 10 October 2008; published 31 March 2009)

\begin{abstract}
We demonstrate that diffusively coupled limit-cycle oscillators on random networks can exhibit various complex dynamical patterns. Reducing the system to a network analog of the complex Ginzburg-Landau equation, we argue that uniform oscillations can be linearly unstable with respect to spontaneous phase modulations due to diffusional coupling - the effect corresponding to the Benjamin-Feir instability in continuous media. Numerical investigations under this instability in random scale-free networks reveal a wealth of complex dynamical regimes, including partial amplitude death, clustering, and chaos. A dynamic mean-field theory explaining different kinds of nonlinear dynamics is constructed.
\end{abstract}

DOI: 10.1103/PhysRevE.79.036214

PACS number(s): 05.45.Xt, 89.75.Fb

Phase oscillators coupled through various network structures have been extensively analyzed as a prototype model of network dynamics [1-4]. In most studies, complete synchronization of all oscillators has been the main focus, though possibilities of more complex dynamics have also been reported $[5,6]$. Coupled phase oscillators are obtained from general coupled limit-cycle oscillators by eliminating amplitude degrees of freedom in the weak-coupling limit [7]. When the coupling is not weak, such phase reduction breaks down and much richer dynamics can be expected.

In this paper, we analyze complex dynamics exhibited by diffusively coupled limit-cycle oscillators on random networks. In continuous media, sufficiently large difference in diffusion constants of oscillating components (e.g., chemical species) can destabilize uniform oscillations and lead to diffusion-induced spatiotemporal chaotic regimes [7], such as those experimentally observed in surface chemical reactions [8]. We argue that diffusional mobility of the components can also lead to the instability and complex dynamics on networks.

Rather than treating a specific model of limit-cycle oscillators, we focus on a network version of the complex Ginzburg-Landau (CGL) equation derived from a general model of diffusively coupled limit-cycle oscillators near the supercritical Hopf bifurcation. Our linear stability analysis based on Laplacian eigenvectors of the network generally shows that the uniformly oscillating solution can become unstable when the analog of the Benjamin-Feir (BF) condition is satisfied. Numerical simulations on random scale-free networks under this condition reveal different kinds of complex dynamical regime. To explain them, an approximate mean-field theory is constructed.

We consider a system of diffusively coupled identical limit-cycle oscillators on random networks consisting of $N$ nodes described by

$$
\dot{\mathbf{X}}_{j}(t)=\mathbf{F}\left(\mathbf{X}_{j}\right)+\mathbf{D} \sum_{k=1}^{N} L_{j k} \mathbf{X}_{k}
$$

Here, $\mathbf{X}_{j}(t)$ represents the state of the oscillator on node $j$ $(j=1, \ldots, N), \mathbf{F}(\mathbf{X})$ specifies the intrinsic dynamics of an oscillator, and the last term takes into account diffusive cou- pling on the network, where $\mathbf{D}$ is a diffusion matrix and $L_{j k}$ is a Laplacian matrix of the network. The network is defined by a symmetric adjacency matrix $A_{j k}$, whose components are 1 if the nodes $j$ and $k$ are connected, and 0 otherwise. The Laplacian matrix is given by $L_{j k}=A_{j k}-k_{j} \delta_{j k}$, with $k_{j}$ $=\sum_{k=1}^{N} A_{j k}$ representing the degree (number of connections) of node $j$. We assume that each oscillator has a stable limitcycle solution $\mathbf{X}^{0}(t)$ in absence of diffusion. A uniformly oscillating solution of the system, $\mathbf{X}_{j}(t) \equiv \mathbf{X}^{0}(t)$ for $\forall j$, always satisfies Eq. (1) because $\sum_{k=1}^{N} L_{j k}=0$ holds, but diffusion may destabilize this solution.

We assume that each oscillator is slightly above the supercritical Hopf bifurcation point and consider a situation where the effect of diffusion is also comparably small. Then, using the standard weakly nonlinear analysis [7], we can reduce Eq. (1) to a network version of the CGL (or Kuramoto-Tsuzuki) equation,

$$
\dot{W}_{j}(t)=\left(1+i c_{0}\right) W_{j}-\left(1+i c_{2}\right)\left|W_{j}\right|^{2} W_{j}+K\left(1+i c_{1}\right) \sum_{k=1}^{N} L_{j k} W_{k} .
$$

Here, $W_{j}(t)$ represents the complex oscillation amplitude of $j$ th oscillator such that $\mathbf{X}_{j}(t)-\mathbf{X}^{(S)} \propto W_{j}(t) \exp \left(i \omega_{0} t\right) \mathbf{U}+$ c.c., where $\mathbf{X}^{(S)}$ is the unstable fixed point, $\omega_{0}$ is the Hopf frequency, and $\mathbf{U}$ is the complex critical eigenvector of the Jacobian matrix of $\mathbf{F}(\mathbf{X})$ at $\mathbf{X}^{(S)}$. Real parameters $c_{0}, c_{1}$, and $c_{2}$, and positive coupling strength $K$ can be determined when $\mathbf{F}(\mathbf{X})$ and $\mathbf{D}$ are explicitly given. Note that if the diffusion constants of all components are equal, i.e., $\mathbf{D}=D \mathbf{I}$, where $\mathbf{I}$ is the identity matrix, we have $c_{1}=0$. Equation (2) has a uniformly oscillating solution, $W_{j}(t) \equiv W^{0}(t):=\exp \left[i\left(c_{0}-c_{2}\right) t\right]$ for $\forall j$.

When $K$ is small enough, each oscillator state is always near the unperturbed limit cycle, so that Eq. (2) can further be reduced to coupled phase oscillators of the form $\dot{\phi}_{j}(t)$ $=\omega-C \sum_{k=1}^{N} L_{j k} \sin \left(\phi_{j}-\phi_{k}+\gamma\right)$, where $\phi_{j}$ is the phase of the oscillator $j, \omega=c_{0}-c_{2}$ is the frequency, $C$ is the rescaled coupling strength, and the coupling phase shift $\gamma$ satisfies $\cos \gamma=\left(1+c_{1} c_{2}\right) / \sqrt{\left(1+c_{1}^{2}\right)\left(1+c_{2}^{2}\right)}$. Recently, it has been shown that this network phase model exhibits coexistence of 
drifting and phase-locked oscillators with stationary phase gradients [5]. In the following, we focus on the case with stronger coupling.

Let us analyze linear stability of the uniform solution. Plugging weakly perturbed solution $W_{j}(t)=W^{0}(t)\{1$ $\left.+\rho_{j}(t)\right\} \exp \left[i \theta_{j}(t)\right]$ into Eq. (2) with $\rho_{j}(t)$ and $\theta_{j}(t)$ being amplitude and phase perturbations, respectively, we obtain the following linearized equations:

$$
\begin{gathered}
\dot{\rho}_{j}(t)=-2 \rho_{j}+K \sum_{k=1}^{N} L_{j k}\left(\rho_{k}-c_{1} \theta_{k}\right), \\
\dot{\theta}_{j}(t)=-2 c_{2} \rho_{j}+K \sum_{k=1}^{N} L_{j k}\left(c_{1} \rho_{k}+\theta_{k}\right) .
\end{gathered}
$$

To proceed, we introduce Laplacian eigenvalues $\Lambda^{(\alpha)}$ and eigenvectors $\phi^{(\alpha)}=\left(\phi_{1}^{(\alpha)}, \ldots, \phi_{N}^{(\alpha)}\right)$ of the Laplacian matrix $L_{j k}$ satisfying $\sum_{k=1}^{N} L_{j k} \phi_{k}^{(\alpha)}=\Lambda^{(\alpha)} \phi_{j}^{(\alpha)}$ for $\alpha=1, \ldots, N$. All eigenvalues are real and nonpositive, and the eigenvectors are mutually orthogonal. We expand the perturbations as $\left(\rho_{j}, \theta_{j}\right)=\sum_{\alpha=1}^{N}\left(\rho^{(\alpha)}, \theta^{(\alpha)}\right) \phi_{j}^{(\alpha)} \exp \left(\lambda^{(\alpha)} t\right)$, where $\rho^{(\alpha)}$ and $\theta^{(\alpha)}$ are expansion coefficients and $\lambda^{(\alpha)}$ is the complex growth rate of $\alpha$ th eigenmode. Then, a characteristic equation $\left\{\lambda^{(\alpha)}\right\}^{2}+2\left\{1-K \Lambda^{(\alpha)}\right\} \lambda^{(\alpha)}-2\left(1+c_{1} c_{2}\right) K \Lambda^{(\alpha)}+\left(1+c_{1}^{2}\right)\left\{K \Lambda^{(\alpha)}\right\}^{2}$ $=0$ is obtained for each eigenmode, which yields

$$
\lambda_{ \pm}^{(\alpha)}=-1+K \Lambda^{(\alpha)} \pm \sqrt{1+2 c_{1} c_{2}\left\{K \Lambda^{(\alpha)}\right\}-c_{1}^{2}\left\{K \Lambda^{(\alpha)}\right\}^{2}} .
$$

When $\operatorname{Re} \lambda_{ \pm}^{(\alpha)}>0$ for some $\alpha$, the $\alpha$ th eigenmode is unstable.

By expanding the upper branch $\lambda_{+}^{(\alpha)}$ of Eq. (4) for small $K \Lambda^{(\alpha)}$, we obtain $\lambda_{+}^{(\alpha)}=\left(1+c_{1} c_{2}\right) K \Lambda^{(\alpha)}+O\left(\left\{K \Lambda^{(\alpha)}\right\}^{2}\right)$. Therefore, $\operatorname{Re} \lambda_{+}^{(\alpha)}$ can be positive when the condition $1+c_{1} c_{2}<0$ is satisfied (note that $\Lambda^{(\alpha)} \leq 0$ ). This is the same as the $\mathrm{BF}$ condition for instability of the uniform solution of the CGL equation in continuous media [7], which also applies to globally coupled and nonlocally coupled CGL oscillators $[9,10]$. Note that the BF condition cannot be satisfied for $c_{1}=0$ and therefore a sufficiently large difference in diffusion constants of the components is necessary. For the instability to actually occur, the discrete Laplacian eigenvalues should exist near the peak of the upper curve given by Eq. (4). As we already know for other coupling schemes $[7,9,10]$, Eq. (2) is expected to exhibit strongly nonlinear behavior once the uniform solution becomes unstable.

As an example of random networks, we use random scalefree networks of size $N=1000$ and mean degrees $\langle k\rangle=20$ generated by the Bárabasi-Albert preferential attachment rule [11]. We fix the parameters $c_{1}=-2$ and $c_{2}=2\left(c_{0}\right.$ can be set to 0 without loss of generality), and vary the coupling strength $K$. Numerical results shown below are for one particular realization of the random network, but similar behavior was observed for other network realizations as well.

Figure 1(a) displays the degree $k_{j}$ of each node vs the node index $j$, where the node indices $\{j\}$ are sorted in decreasing order of their degrees $\left\{k_{j}\right\}$ so that inequalities $k_{1}$ $\geq k_{2} \geq \cdots \geq k_{N}$ hold. We use this ordering as a useful way to visualize the complex dynamics on the network throughout our analysis. Figure 1(b) shows the Laplacian eigenvalues (a)

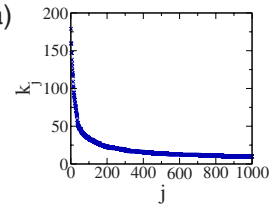

(b)

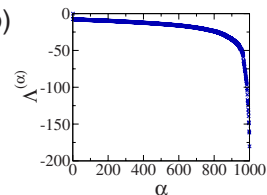

(c)

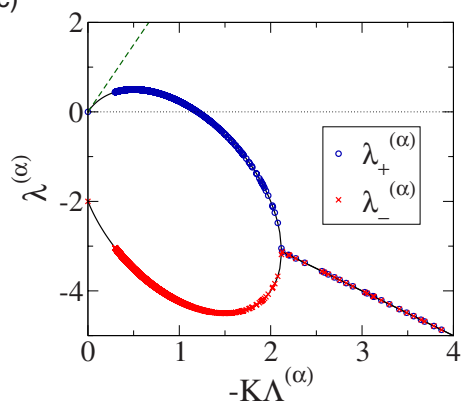

FIG. 1. (Color online) (a) Degree $k_{j}$ and (b) Laplacian eigenvalue $\Lambda^{(\alpha)}$ of the scale-free network used in numerical simulations. (c) Linear growth rates of perturbations $\operatorname{Re} \lambda_{+}^{(\alpha)}$ plotted as functions of $-K \Lambda^{(\alpha)}$. Dashed green line shows the slope $-\left(1+c_{1} c_{2}\right)$ of the upper branch at the origin.

$\Lambda^{(\alpha)}$ of the same network. The eigenvalue indices $\{\alpha\}$ are also sorted in decreasing order of the eigenvalues such that $0=\Lambda^{(1)}>\Lambda^{(2)}>\cdots>\Lambda^{(N)}$ hold.

Figure $1(\mathrm{c})$ plots growth rates of the perturbations $\operatorname{Re} \lambda_{ \pm}^{(\alpha)}$ obtained by the linear stability analysis as functions of $-K \Lambda^{(\alpha)}$ at $K=0.04$. The actual growth rates are distributed discretely on the curves given by Eq. (4). We can see that the growth rates on the upper branch $\operatorname{Re} \lambda_{+}^{(\alpha)}$ can become positive when the coupling strength $K$ is in an appropriate range, indicating that the uniform solution can undergo a diffusioninduced instability.

To investigate nonlinear dynamics after the instability, we have performed numerical simulations of Eq. (2) with slightly perturbed uniform solutions as initial conditions. When $K$ is very small $(K<0.001)$, no oscillator deviates largely from the unperturbed limit-cycle orbit $W^{(0)}(t)$, so that the reduced phase model is valid. The coupling phase shift is given by $\gamma=\arccos (-3 / 5) \simeq-2.21$, which is repulsive because $|\gamma|>\pi / 2$ [7]. Therefore, the oscillators do not synchronize but rotate incoherently. When $K$ is very large $(K$ $>0.165)$, there exist no discrete growth rates on the positive part of the upper curve of Fig. 1(c), so that the uniform solution remains stable even if the BF condition is satisfied.

Between these limits, we have found three characteristic steady dynamical regimes as shown in Fig. 2, where snapshots of the amplitude profile $\left|W_{j}\right|$ and the distribution of $W_{j}$ on the complex plane are displayed for three values of the coupling strength, $K=0.02, K=0.04$, and $K=0.08$.

(i) Partial amplitude death [Figs. 2(a) and 2(d)]. When $0.006<K<0.028$, a group of oscillators with small node indices (i.e., with large degrees) stops rotation and stays near the origin of the complex plane while other oscillators are rotating around circular orbits incoherently, with a rather sharp but smooth transition between the two groups.

(ii) Chaos [Figs. 2(b) and 2(e)]. When $0.028<K<0.078$, the oscillators are roughly separated into three groups. In the first group, oscillators take approximately constant amplitudes near 0.5, which corresponds to the central cluster on the complex plane. Amplitudes of oscillators in the second group are strongly scattered and evolve chaotically, but their envelope still forms smooth curves. This group corresponds to the intermediate scattered oscillator states on the complex 
(a)

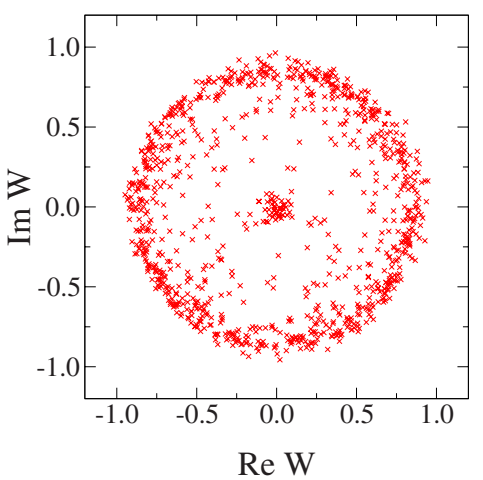

(d)

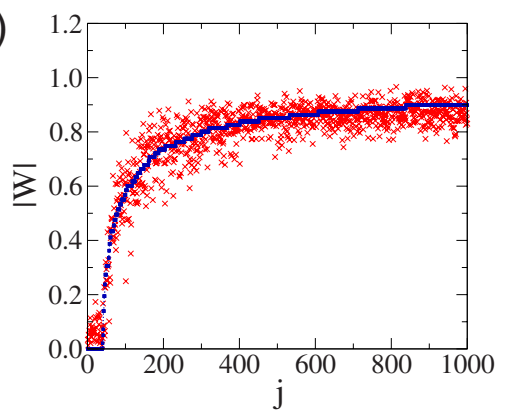

(b)

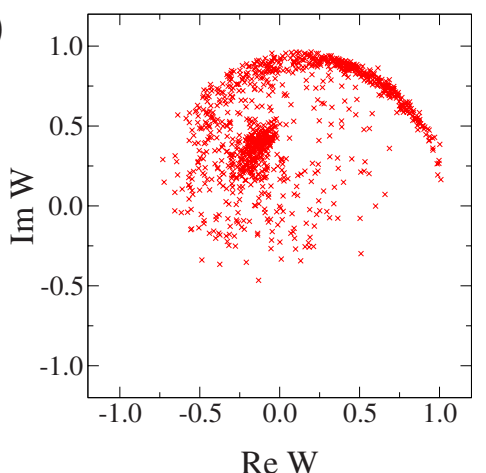

(e)

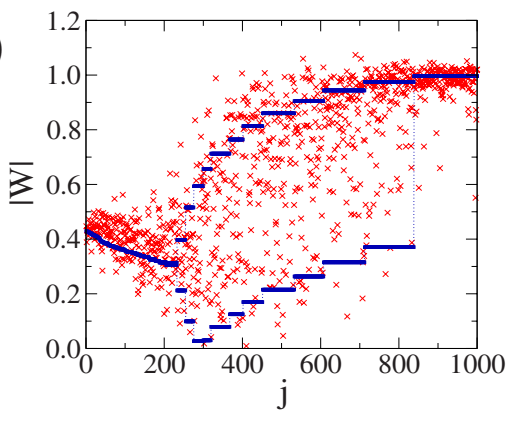

(c)

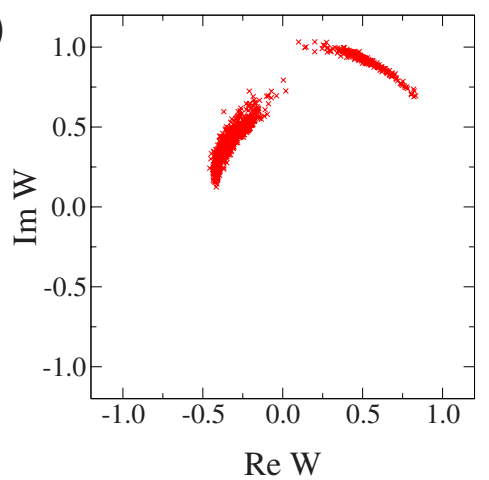

(f)

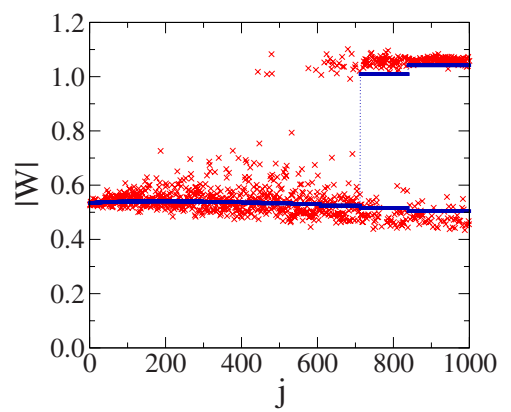

FIG. 2. (Color online) [(a)-(c)] Snapshots of the complex amplitude $W_{j}$ on the complex plane. [(d)-(f)] Snapshots of the amplitude $\left|W_{j}\right|$ vs the node index $j$. Coupling strength is $K=0.02$ for (a) and (d), $K=0.04$ for (b) and (e), and $K=0.08$ for (c) and (f). Node indices are sorted in decreasing order of their degrees $\left\{k_{j}\right\}$ so that inequalities $k_{1} \geq k_{2} \geq \cdots \geq k_{N}$ hold. Solid blue curves in (d)-(f) are predictions of the mean-field theory.

plane. The oscillators in the last group again take constant amplitudes near 1 , which correspond to the oscillator states elongated along the unit circle on the complex plane. The largest Lyapunov exponent of the system is positive in this regime.

(iii) Clustering [Figs. 2(c) and 2(f)]. When $0.078<K$ $<0.164$, phase relations among the oscillators are frozen and the whole system exhibits a rigid constant rotation. For relatively small values of $K(K<0.12)$, the oscillators with small degrees split into two groups with two distinct amplitudes; i.e., they exhibit a two-cluster state. As $K$ becomes larger, the two clusters gradually approach each other and, at relatively large $K(K>0.14)$, the two clusters merge to a single cluster but still with phase scattering.

Transitions between the above dynamical regimes occur abruptly and are clearly detectable, whereas the change in the dynamics within each regime, e.g., transformation from twocluster to one-cluster states, occurs gradually with $K$.

To explain the observed dynamical patterns, we employ the mean-field approximation, valid for large random networks with strong diffusive mixing. It has been used in analyzing network-based epidemic spreading models [12], coupled phase oscillators [3,5], and also network Turing patterns [13]. A crucial point here is that we consider not only static but also dynamic mean fields that oscillate periodically with time.

Introducing a complex local field $h_{j}(t)=\sum_{k=1}^{N} A_{j k} W_{k}(t)$, the diffusion term in Eq. (2) can be written as $\sum_{k=1}^{N} L_{j k} W_{k}=h_{j}(t)$ $-k_{j} W_{j}$. We approximate this local field as

$$
h_{j}(t) \simeq k_{j} H(t), \quad H(t)=\sum_{j=1}^{N} \frac{k_{j}}{k_{\mathrm{tot}}} W_{j}(t),
$$

where $k_{\mathrm{tot}}=\sum_{j=1}^{N} k_{j}$ and $H(t)$ is a degree-weighted global mean field over the network $[3,12,13]$. Thus, we ignore detailed connections of the network and retain only the degrees. Equation (2) is then approximated as

$$
\begin{aligned}
\dot{W}_{j}(t)= & \left(1+i c_{0}\right) W_{j}-\left(1+i c_{2}\right)\left|W_{j}\right|^{2} W_{j} \\
& +k_{j} K\left(1+i c_{1}\right)\left\{H(t)-W_{j}\right\},
\end{aligned}
$$

which describes independent CGL oscillators coupled to a global mean field $H(t)$. The effective coupling strength of each oscillator to $H(t)$ is given by $k_{j} K$, and thus depends on the node degree $k_{j}$.

In Figs. 3(a), 3(d), and 3(g), time sequences of the global mean field $H(t)$ obtained numerically for the three cases in Fig. 2 are shown. $H(t)$ almost vanishes at $K=0.02$, whereas it oscillates sinusoidally at $K=0.04$ and $K=0.08$. We can thus approximate $H(t)$ in these regimes as $H(t)=B \exp (i \Omega t)$, where $B$ and $\Omega$ denote amplitude and frequency of the periodic sinusoidal oscillation, which reasonably fit the numerical data as shown in the figures. Similar sinusoidal-field approximation has been used in the analysis of collective dynamics of globally coupled CGL oscillators [9], but degree inhomogeneity in networks essentially changes the results. Precisely speaking, in the chaotic regime, $H(t)$ is only approximately sinusoidal and can be more complex, e.g., qua- 
(a)

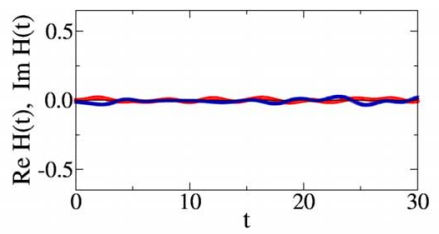

(b)

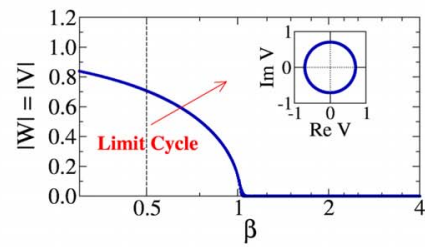

(d)

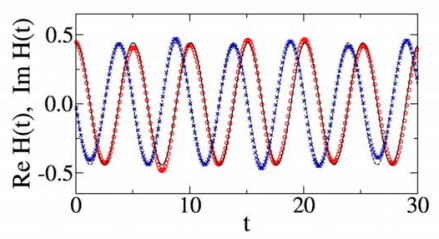

(e)

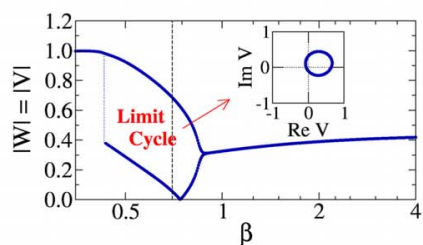

(g)

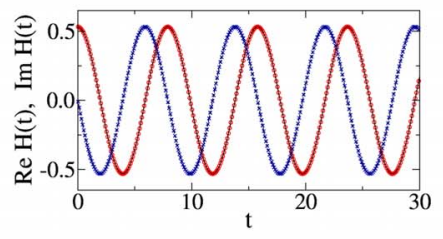

(h)

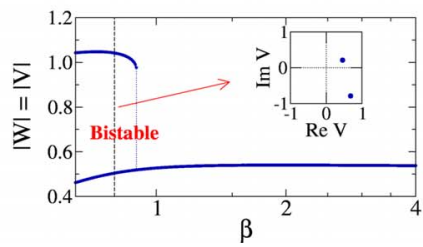

(c)

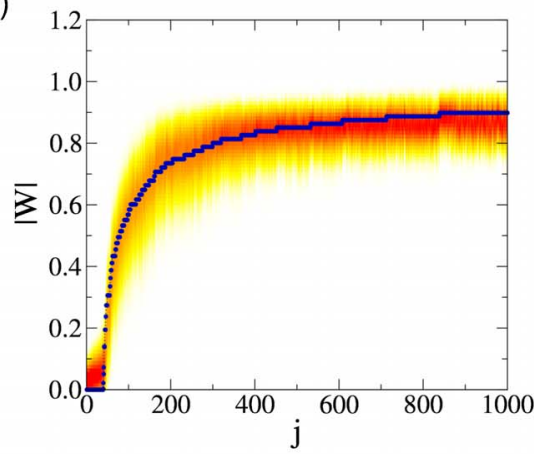

(f)

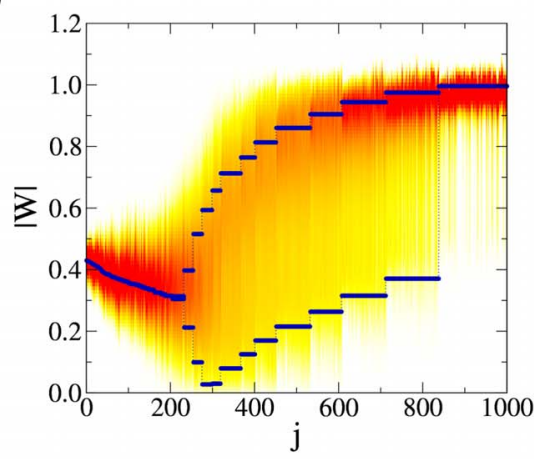

(i)

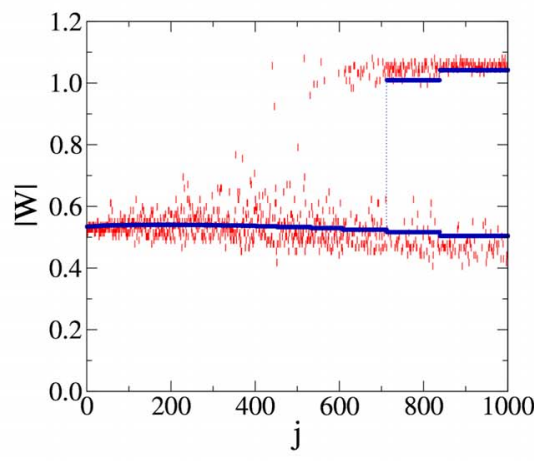

FIG. 3. (Color online) [(a), (d), and $(\mathrm{g})$ ] Evolution of real (red circles) and imaginary parts (blue crosses) of the global mean field $H(t)$ and fitting by $B \exp (i \Omega t)$. $[(\mathrm{b}),(\mathrm{e})$, and (h)] Bifurcation diagrams of the sinusoidally driven oscillator. Insets show limit-cycle orbits or fixed points of $V(t)$ at the parameter values indicated by broken vertical lines. [(c), (f), and (i)] Probability density functions of $\left|W_{j}\right|$ compared with the mean-field approximation (yellow or light gray represents lower density, and red or dark gray represents higher density, with white representing zero). Solid blue curves represent the maximal and minimal values of the complex amplitude $\left|W_{j}\right|$ under the mean-field approximation. Parameters are $K=0.02$ and $B=0$ for (a)-(c); $K=0.04, \quad B=0.442$, and $\Omega=-1.25$ for $(\mathrm{d})-(\mathrm{f})$; and $K$ $=0.08, B=0.532$, and $\Omega=-0.796$ for (g)-(i). siperiodic for some other values of $K$ (as also known in the case of global coupling [9]), but we focus on the simplest sinusoidal case here. In the clustering regime, $H(t)$ is always strictly sinusoidal.

Moving to a rotating frame by introducing $W(t)$ $=V(t) \exp (i \Omega t)$, we obtain an autonomous equation for $V(t)$ as $\quad \dot{V}(t)=\left[1+i\left(c_{0}-\Omega\right)\right] V-\left(1+i c_{2}\right)|V|^{2} V+\beta\left(1+i c_{1}\right)(B-V)$. Here we dropped the index $j$, because all oscillators obey the same dynamics, and defined $\beta=\beta(j)=k_{j} K$, which plays the role of a bifurcation parameter. The dependence of the oscillator dynamics on the node index $j$ enters only through $\beta$.

Figures 3(b), 3(e), and 3(h) display the bifurcation diagrams of the above equation as functions of the control parameter $\beta$, where the maximal and the minimal values of $|W|=|V|$ are plotted using $B$ and $\Omega$ estimated numerically in Figs. 3(a), 3(d), and 3(g). Depending on the values of $B, \Omega$, and $\beta$, the equation exhibits a symmetric limit cycle, an asymmetric limit cycle, and one or two fixed points [14].
Now, using the relation between the bifurcation parameter and the node degree, $\beta=\beta(j)=K k_{j}$, we can map the bifurcation diagrams onto actual amplitude patterns in the network. The solid curves in Figs. 2(d)-2(f) are the maximal and minimal values of $\left|W_{j}\right|=\left|V_{j}\right|$, which fit the envelopes of the oscillator dynamics reasonably well. Figures 3(c), 3(f), and 3(i) compare the numerical probability density functions of the amplitude with these curves, showing good agreement. In particular, the condition for an oscillator to fall in the amplitude death state in regime (i) can be obtained analytically by linear stability analysis of the fixed point $V_{j}=0$ with $B=0$. This yields $k_{j}>1 / K$, which also agrees well with the numerical data. Thus, the complex network dynamics in our model can be well understood through the mean-field approximation [15].

Summarizing, we have investigated diffusion-induced instability and resulting complex dynamics exhibited by limitcycle oscillators on random networks. Under the mean-field 
approximation, the observed inhomogeneous dynamical patterns can be interpreted as a mixture of various limit cycles and fixed points, which is reminiscent of the "chimera" states found in nonlocally coupled oscillators $[5,16]$. In the present case, however, the degree inhomogeneity of the network essentially determines the dynamics of each oscillator.

Dynamical systems coupled through various networks are ubiquitous structures in the real world, ranging from neuronal circuits in the brain to various engineering problems, such as sensor networks and power grids (see $[1,4])$. The fact that complex dynamical patterns can spontaneously emerge in random oscillator networks may be of fundamental importance in understanding the behavior and functions of such systems.

Financial support of the Volkswagen Foundation (Germany) and the MEXT, Japan (Kakenhi Grant No. 19762053) is gratefully acknowledged.
[1] S. H. Strogatz, Nature (London) 410, 268 (2001).

[2] Y. Moreno and A. F. Pacheco, Europhys. Lett. 68, 603 (2004).

[3] T. Ichinomiya, Phys. Rev. E 70, 026116 (2004).

[4] A. Arenas, A. Díaz-Guilera, J. Kurths, Y. Moreno, and C. Zhou, Phys. Rep. 469, 93 (2008).

[5] T.-W. Ko and G. B. Ermentrout, Phys. Rev. E 78, 016203 (2008).

[6] S. Gil and A. S. Mikhailov, Phys. Rev. E 79, 026219 (2009).

[7] Y. Kuramoto, Chemical Oscillations, Waves, and Turbulence (Springer, Berlin, 1984).

[8] M. Kim, M. Bertram, M. Pollmann, A. von Oertzen, A. S. Mikhailov, H. H. Rotermund, and G. Ertl, Science 292, 1357 (2001).

[9] V. Hakim and W.-J. Rappel, Phys. Rev. A 46, R7347 (1992); N. Nakagawa and Y. Kuramoto, Prog. Theor. Phys. 89, 313 (1993); M.-L. Chabanol, V. Hakim, and W.-J. Rappel, Physica D 103, 273 (1997); M. Banaji and P. Glendinning, Phys. Lett. A 251, 297 (1999).

[10] Y. Kuramoto, Prog. Theor. Phys. 94, 321 (1995); Y. Kuramoto and H. Nakao, Phys. Rev. Lett. 76, 4352 (1996); H. Nakao, Chaos 9, 902 (1999).

[11] R. Albert and A.-L. Barabási, Rev. Mod. Phys. 74, 47 (2002).

[12] R. Pastor-Satorras and A. Vespignani, Phys. Rev. Lett. 86, 3200 (2001).

[13] H. Nakao and A. S. Mikhailov, e-print arXiv:0807.1230.

[14] In [9], it is reported that the asymmetric limit cycle can coexist with the fixed points in a certain parameter region, where the transition with hysteresis occurs via a saddle-node bifurcation of the fixed points followed by a homoclinic or Hopf bifurcation of the limit cycle. In the case of Fig. 3(e), the transition near $\beta=0.435$ occurs by a saddle node on invariant circle (SNIC) bifurcation without hysteresis, and coexistence of fixed points with the asymmetric limit cycle does not take place. Note that coexistence of multiple attractors in the dynamics of individual oscillators, which actually occurs in the two-cluster state [Fig. 3(h)], does not affect our argument as long as the global mean field is approximately sinusoidal.

[15] Though we do not give details in the present paper, we can further develop a self-consistency analysis for the sinusoidal global mean field $H(t)$ to determine $B$ and $\Omega$ from the condition that the $H(t)$ imposed on Eq. (6) coincide with the $H(t)$ resulting from Eq. (5). This gives good agreement with direct numerical results for $0 \leq K<0.05$, where $H(t)$ vanishes or oscillates sinusoidally and each individual oscillator has a single attractor. For larger values of $K$ where $H(t)$ is not sinusoidal or some of the individual oscillators have multiple attractors (e.g., bistable fixed points), such a simple sinusoidal self-consistency analysis fails. See Chabanol et al. [9] for elaborate analysis of the globally coupled CGL oscillators.

[16] Y. Kuramoto and D. Battogtokh, Nonlinear Phenom. Complex Syst. (Dordrecht, Neth.) 5, 380 (2002); S.-i. Shima and Y. Kuramoto, Phys. Rev. E 69, 036213 (2004); D. M. Abrams and S. H. Strogatz, Phys. Rev. Lett. 93, 174102 (2004). 